\title{
CONHECIMENTO SOBRE PLANTAS TÓXICAS EM ESCOLAS MUNICIPAIS DE CODÓ-MA, BRASIL
}

Jouden Lima Conceição ${ }^{1}$, Edinones Holanda da Silva ${ }^{1}$, William dos Santos Ribeiro ${ }^{2}$, Aretha Franklin Guimarães ${ }^{2}$, Eduardo Oliveira Silva ${ }^{2,3}$

1 Curso de Licenciatura em Ciências Naturais, Universidade Federal do Maranhão, UFMA/Codó, Maranhão, Brasil.

2,3 Programa de Pós-Graduação em Botânica Aplicada-Doutorado, UFLA/Lavras, Minas Gerais, Brasil

3 Prof. Assistente da Universidade Federal do Maranhão, UFMA/Codó, Maranhão,

Brasil. Email: eoliveira12@yahoo.com.br

Recebido em: 22/09/2018 - Aprovado em: 23/11/2018 - Publicado em: 03/12/2018 DOI: 10.18677/EnciBio_2018B82

\begin{abstract}
Nosso objetivo foi coletar informações sobre plantas tóxicas nas escolas públicas e acessar informações sobre o conhecimento dos alunos sobre o tema. Realizamos esse trabalho em 21 escolas municipais de ensino público em Codó-MA. Em 12 das escolas, encontramos plantas tóxicas. Foram identificadas cinco famílias botânicas e 13 espécies de plantas tóxicas. As informações coletadas foram controversas: alguns dos entrevistadas só conheciam as plantas pelo nome, mas não conseguiam identificá-las. Nesse sentido, é importante elaborar projetos com essas escolas que incluam no currículo escolar temas que possam iluminar o conhecimento sobre plantas tóxicas com o objetivo de prevenir acidentes e melhorar o entendimento da população sobre sua flora local.
\end{abstract}

RESUMO

PALAVRAS-CHAVE: intoxicação, plantas ornamentais, prevenção

\section{KNOWLEDGE ON TOXIC PLANTS IN MUNICIPAL SCHOOLS OF CODÓ-MA,} BRAZIL

\begin{abstract}
Our aim was to collect information about toxic plants in public schools and to access information about the students knowledge on the theme. We carried this work in 21 municipal public schools in Codó-MA. In 12 of the schools we found toxic plants. We identified five botanical families and thirteen species of toxic plants. The information we collected were controversial: some of interviewed only knew the plants by name, but weren't able to identify them. In this sense, it is important to elaborate projects with these schools that include in the scholar curriculum themes that shed light on the knowledge about toxic plants with the purpose to prevent accidents and to enhance the populations understanding about their local flora.
\end{abstract}

KEYWORDS: Ornamental plants, prevention, intoxication 


\section{INTRODUÇÃO}

O contato com o meio ambiente tem se tornado cada vez menor, ocasionado pelo processo de urbanização, ocorrendo sempre mais acelerado e distanciando a população das áreas naturais (BAMPI et al., 2014). Como elemento do ambiente natural, as plantas desempenham importante papel na natureza, representando, também, fonte de alimento e produtos para o homem. No paisagismo e ornamentação de ambientes, as plantas são fundamentais, pelo que atendem às necessidades estéticas e de conforto ambiental (BIONDI et al., 2008).

Nos últimos anos, em razão da promoção de uma conscientização ambiental, a sociedade tem sido estimulada a cultivar espécies vegetais, inclusive em ambientes escolares, muitas vezes com intuitos ornamentais (ALVES et al., 2016). Entretanto, é necessário ter conhecimento de quais espécies são mais apropriadas para cada ambiente e local, principalmente em áreas onde a presença de crianças é constante, como nas escolas e creches, já que algumas plantas representam perigo por ter estruturas como espinhos, tricomas e componentes químicos tóxicos, o que podem ocasionar acidentes quando tocadas ou ingeridas.

Plantas tóxicas podem ser definidas como todo e qualquer vegetal que em contato com os seres humanos ou animais, causam danos à saúde, podendo levá-los a óbito. Os principais agentes tóxicos encontrados são oxalato de cálcio, saponinas, alcalóides beladonados (atropina, escopolamina e hioscina), glicosídeos cardiotóxicos e toxalbumina (ricina), sendo que a maioria ocorre em todas as partes das plantas (ARAÚJO et al., 2018).

Por ser um ambiente público, a escola deve preocupar-se em realizar um estudo específico sobre as espécies que serão utilizadas como ornamentais, uma vez que esses dados podem auxiliar no manuseio e no planejamento do cultivo destas, além de evitar o plantio de vegetais que podem provocar acidentes como as espécies que causam intoxicações (BRAGA et al., 2017).

A ocorrência de casos de intoxicação por plantas em crianças ocorre pelo fato destas estarem continuamente descobrindo o ambiente, o que influi nelas a atração pelas cores fortes e vibrantes das folhas, flores e frutos, aguçando assim a sua curiosidade, fazendo com que elas tenham contato com as mesmas, não imaginando o perigo que alguns desses vegetais possam oferecer (BRAGA, 2017). Segundo Sistema Nacional de Informações Tóxico-Farmacológica (SINITOX, 2016), foram registrados 356 casos de intoxicação por plantas em zonas urbanas, e cerca de 232 casos somente na faixa etária entre 1 a 4 anos.

Para Alves et al. (2016), estudantes do ensino fundamental, além de crianças em idade escolar, não possuem acesso a informações corretas e concretas sobre essa problemática, e chama a atenção para a importância da educação em saúde como uma forma de conscientizar a população sobre esses riscos. Assim, esse estudo objetivou listar as plantas tóxicas cultivadas em escolas públicas na cidade de Codó-MA e verificar o conhecimento dos agentes escolares sobre a toxicidade dessas plantas e sua representatividade no ambiente escolar.

\section{MATERIAL E MÉTODOS}

\section{Área de estudo}

O município de Codó ( $4^{\circ} 25^{\prime} 05^{\prime}$ 'Sul, $43^{\circ} 52^{\prime} 57^{\prime}$ 'Oeste), está localizado na mesorregião Leste Maranhense e na Microrregião de Codó, possui área territorial de $4.382,49 \mathrm{~km}^{2}$. De acordo com o censo de 2017, ocupa a sexta posição na lista dos municípios mais populosos do estado com 120.810 habitantes (IBGE, 2017). 
No âmbito municipal, Codó conta com 217 escolas, no qual a maior parte, encontra-se na zona rural, com 158 escolas e somente 59 na zona urbana.

\section{Coleta de dados}

Primeiramente, foi apresentado aos responsáveis pelos estabelecimentos escolares uma carta contendo os objetivos e as etapas do projeto, solicitando a permissão para a realização das entrevistas com diretores e funcionários, que concordaram participar voluntariamente da pesquisa. Esta foi desenvolvida no período de agosto a novembro de 2016, em 21 escolas da rede pública municipal, de cinco bairros (São Antônio, São Francisco, São José, São Raimundo e São Sebastião), na cidade de Codó-MA (Figura 1).

A proposta de trabalhar nas escolas surgiu pelo fato das mesmas serem frequentadas principalmente por crianças entre 5 a 9 anos, sendo que esta idade é mais vulnerável a ocorrência de acidentes principalmente por intoxicação.

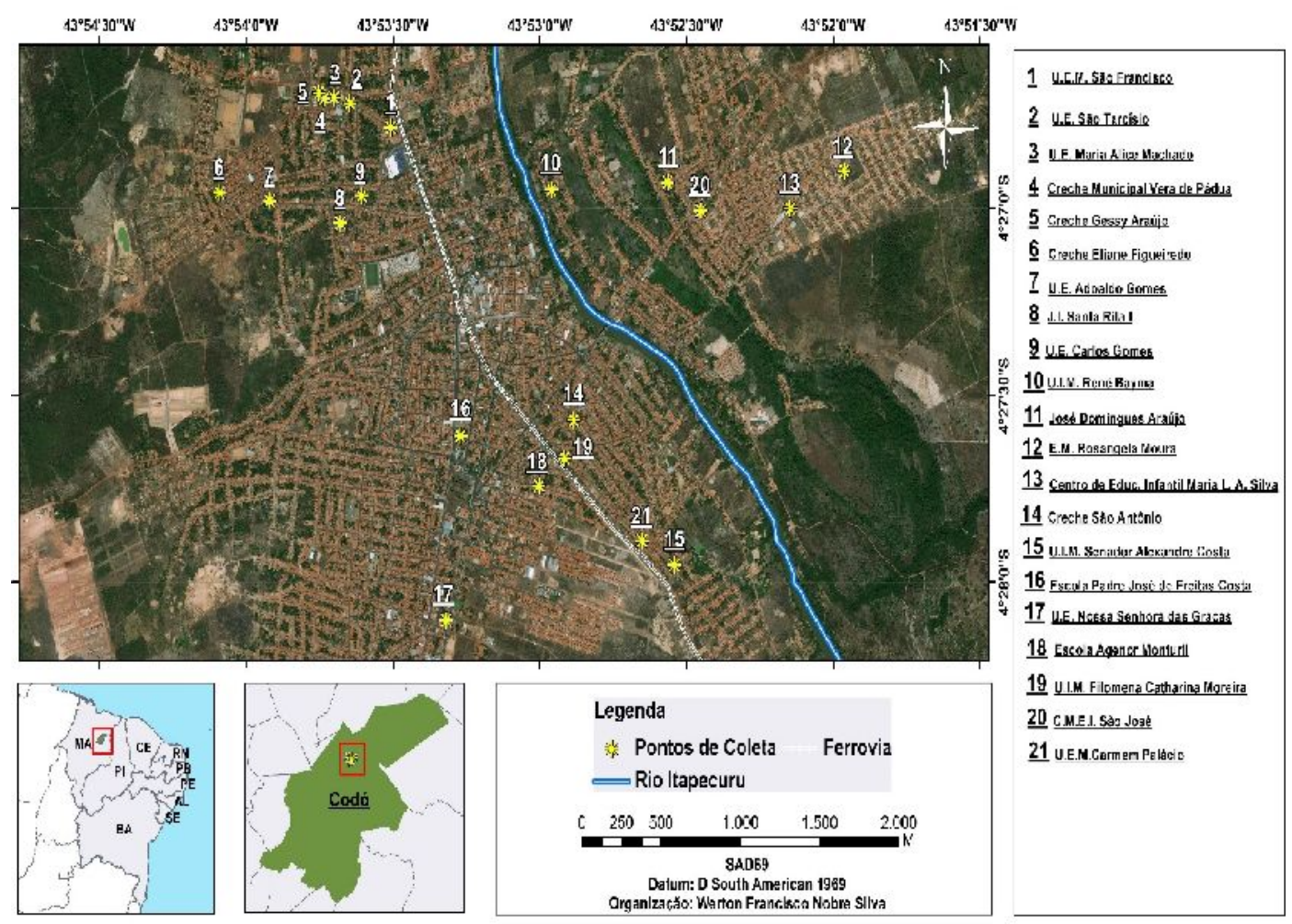

FIGURA 1: Localização das Escolas Municipais pesquisadas em Codó MA. Fonte: ArcGis 9.3; Google Earth 2017; Organização SILVA, W.N.F. 2017

Em seguida, foram visitadas 21 escolas nos bairros selecionados. No bairro São Antônio foram visitadas as escolas U.E.M Carmem Palácio largo, Creche São Antônio, U.I.M. Filomena Catharina Moreira e U.I.M. Senador Alexandre Costa; no Bairro São Francisco: C.M.E.I. Santa Filomena, Colégio Municipal São Francisco, Creche Eliane Figueiredo, Creche Gessy Araújo, Creche Municipal Vera de Pádua, E.M. São Tarcísio; U.E. Adoaldo Gomes, U.E. Carlos Gomes e U.E. Maria Alice Machado; bairro São José: U.I.M. Renê Bayma; bairro São Raimundo: E.I. E.M. Rosangela Moura, Centro de Educação Infantil Maria Luiza Araújo Silva, U.E.M. José 
Domingues Araújo e C.M.E. São José; e no bairro São Sebastião: Escola Agenor Montoril, Escolas Padre José de Freitas Costa e U. E. Nossa Senhora das Graças.

Em todas essas escolas, foram realizadas observações diretas em seus espaços físicos para o levantamento das plantas ornamentais. Na ocasião, ocorreu também a aplicação de dois questionários, direcionados aos gestores, professores, vigilantes e auxiliar operacional de serviço, para obtenção de informações acerca do conhecimento das plantas ornamentais e tóxicas presentes nas escolas.

As espécies encontradas foram reconhecidas no local, pelo nome vulgar dado pelos informantes, fotografadas, e posteriormente identificadas taxonomicamente. A identificação das espécies foi realizada, com auxílio de literaturas especializadas como catálogos e livros sobre o tema (RANGEL, 2000; MATOS et al., 2011; BOCHNER et al., 2013); através de chaves de identificação taxonômica e consulta nas bases de dados do The Plant List (2018) e Tropicos (2018). O Sistema de Classificação Botânica seguiu o Angiosperm Phylogeny Group IV- APG IV (2016). Os dados foram tabulados em Planilha Excel (2010) e organizados em ordem alfabética das famílias as quais as espécies pertenciam.

\section{RESULTADOS E DISCUSSÃO}

\section{Conhecimento sobre plantas tóxicas}

Foram entrevistadas 48 pessoas, sendo 36 do sexo feminino (75\%) e 12 do sexo masculino (25\%). Os questionários foram aplicados aos professores das escolas, que correspondem a $46 \%$ dos entrevistados, seguido dos diretores com $29 \%$, vigilantes $21 \%$ e zeladores com $4 \%$. Observa-se que os professores foram a maioria dos entrevistados, visto que os mesmos, através de suas funções, têm a possibilidade de conhecer e trabalhar melhor a temática sobre plantas tóxicas com os alunos em sala de aula. Alves et al. (2016) ressaltam a importância da inclusão de professores e coordenadores em projetos de extensão que envolvam essas temáticas, visto que a maior parte das crianças encontra-se, muitas vezes, em vida escolar sob responsabilidade desses profissionais.

Ao perguntar aos gestores quem é o responsável pelo cuidado das plantas, $71,42 \%$ dos entrevistados responderam que são os vigias da escola; $7,14 \%$ relataram serem os professores; $7,14 \%$ responderam que são os zeladores e $14,28 \%$ responderam que seriam a secretaria de infraestrutura, que cuida das plantas existentes nas escolas. Quando perguntado para os funcionários responsáveis pelas plantas, quais os principais motivos que levaram a escolhas e permanência das mesmas na ornamentação das escolas, $43 \%$ responderam que os critérios adotados foram as características das folhas; $16 \%$ pela beleza ornamental; $13 \%$ afirmaram que foram escolhidas pela cor; $12 \%$ pelas características das flores; $8 \%$ pelo tamanho, e $8 \%$ outros motivos.

Biondi et al. (2008), defendem que a intervenção paisagística nos pátios das escolas deveria ser planejada não só para atender as necessidades estéticas e de conforto ambiental dos usuários, mais também para servir de ferramenta para as práticas educativas, utilizando esses instrumentos para ilustrar os conhecimentos teóricos relativos à saúde humana e à conservação da natureza.

Das 21 escolas pesquisadas, em 9 não foram encontradas nenhum vegetal tóxico, porém em 12 foram constatadas a presença de plantas tóxicas nos jardins e arredores do ambiente escolar ( quadro 1). 
QUADRO 1. Quantidade de espécies tóxicas por escolas no município de Codó-MA.

\begin{tabular}{|c|c|c|}
\hline Escola & Espécie & Quantidade \\
\hline C.M.E.I Santo Antônio & $\begin{array}{l}\text { Catharanthus roseus (L.) G.Don } \\
\text { Euphorbia tirucalli L. } \\
\text { Sansevieria trifasciata Prain }\end{array}$ & 3 \\
\hline $\begin{array}{l}\text { Creche Municipal Vera de } \\
\text { Pádua }\end{array}$ & $\begin{array}{l}\text { Jatropha gossypiifolia L. } \\
\text { Sansevieria trifasciata Prain } \\
\text { Tradescantia spathacea Sw. }\end{array}$ & 3 \\
\hline $\begin{array}{l}\text { Colégio Municipal São } \\
\text { Francisco }\end{array}$ & $\begin{array}{l}\text { Calotropis procera (Aiton) W.T. Aiton } \\
\text { Manihot esculenta Crantz } \\
\text { Ricinus communis L. }\end{array}$ & 3 \\
\hline C.M.E.I. São José & $\begin{array}{l}\text { Dieffenbachia seguine (Jacq.) Schott } \\
\text { Tradescantia spathacea Sw. }\end{array}$ & 2 \\
\hline C.M.E.I Santa Filomena & Sansevieria trifasciata Prain & 2 \\
\hline E.M. Rosangela Moura & $\begin{array}{l}\text { Anthurium andraeanum Liden } \\
\text { Catharanthus roseus (L.) G.Don } \\
\text { Codiaeum variegatum (L.) Rumph. ex A.Juss } \\
\text { Dieffenbachia seguine (Jacq.) Schott } \\
\text { Sansevieria trifasciata. Prain } \\
\text { Tradescantia spathacea Sw. }\end{array}$ & 6 \\
\hline $\begin{array}{l}\text { Escola Padre José de } \\
\text { Freitas Costa }\end{array}$ & Sansevieria trifasciata Prain & 1 \\
\hline U.E. Adoaldo Gomes & $\begin{array}{l}\text { Dieffenbachia seguine (Jacq.) Schott } \\
\text { Jatropha gossypiifolia L. } \\
\text { Sansevieria trifasciata Prain } \\
\text { Catharanthus roseus (L.) G.Don }\end{array}$ & 4 \\
\hline U.E.Maria Alice Machado & $\begin{array}{l}\text { Sansevieria trifasciata. Prain } \\
\text { Manihot esculenta Crantz }\end{array}$ & 2 \\
\hline $\begin{array}{l}\text { U.E.M. José Domingues } \\
\text { Araújo }\end{array}$ & $\begin{array}{l}\text { Diefenbachia seguine (Jacq.) Schott } \\
\text { Catharanthus roseus (L.) G.Don } \\
\text { Sansevieria trifasciata Prain } \\
\text { Calotropis procera (Aiton) W.T. Aiton } \\
\text { Codiaeum variegatum (L.) Rumph. ex A.Juss }\end{array}$ & 5 \\
\hline U.I.M. Renê Bayma & $\begin{array}{l}\text { Thevetia peruviana (Pers.) K.Schum. } \\
\text { Sansevieria trifasciata Prain }\end{array}$ & 2 \\
\hline $\begin{array}{l}\text { U.I.M. Senador Alexandre } \\
\text { Costa }\end{array}$ & Jatropha gossypiifolia L. & 1 \\
\hline
\end{tabular}

A Escola Municipal Rosângela Moura, localizada no bairro São Raimundo, apresentou maior número de plantas tóxicas (seis espécies). A escola possui cinco salas de aula de primeiro ao quinto ano, com funcionamento nos turnos matutino e vespertino e atende um total de 365 alunos. A escola não possui jardim sendo que as plantas estão presentes no pátio e arredores da escola. A segunda escola com mais plantas tóxicas (cinco espécies) foi a Unidade Escolar Municipal José Domingues Araújo, também situada no bairro são Raimundo. A escola possui sete salas de aula com funcionamento nos três turnos com ensino do primeiro ao quinto ano e a 
modalidade EJA, com primeira e segunda etapa noturno. As plantas estão presentes nos arredores e em jarros no pátio.

A terceira escola com maior número de espécies foi a escola Adoaldo Gomes, localizada no bairro São Francisco (quatro espécies). A escola tem seis salas de aulas atendendo 410 alunos. As plantas encontram-se no jardim e arredores. Nas demais escolas foram registradas menos de quatro espécies.

Pode-se perceber que nesses espaços, as espécies ornamentais nem sempre estão plantadas nos locais mais apropriados. Em apenas cinco delas foram encontrados jardins destinados às plantas, estando estas em local de fácil acesso para as crianças como também observam Silva et al. (2017). Segundo Rodriguez et al. (2014) as plantas tóxicas geralmente são plantas de vasos ou plantas daninhas, adquiridas pelas pessoas pelo seu caráter ornamental, porém sem saberem dos riscos e poder de intoxicação que as mesmas possuem.

Quando perguntado para os entrevistados se conheciam ou se já ouviram falar sobre alguma planta tóxica, $62,5 \%$ responderam que sim, enquanto $37,5 \%$ responderam não conhecer. As plantas mais citadas foram: comigo-ninguém-pode (Dieffenbachia seguine), seguida de espada-de-são-jorge (Sansevieria trifasciata), cansanção (Urtica dioica), chapéu-de-napoleão (Thevetia peruviana), mamona (Ricinus communis), cachorro-pelado (Euphorbia tirucalli) e pinhão-roxo (Jatropha Gossypiipholia). Porém, quando perguntados sobre a ocorrência de plantas tóxicas nas escolas, 14,5\% afirmaram que não; $29,1 \%$ afirmaram que há presença de planta tóxica na escola, enquanto $56,4 \%$ disseram não saber da existência de plantas tóxicas dentro da escola.

De acordo com as informações obtidas sobre os riscos que as plantas tóxicas oferecem, 54\% dos informantes afirmaram que conheciam, e $46 \%$ afirmaram que não conheciam os riscos. No Brasil, os acidentes com plantas tóxicas são frequentes, pois a maior parte da população não possui um conhecimento, ou apenas superficial sobre o assunto, sendo que os acidentes mais comuns ocorrem com a planta "comigo-ninguém-pode" ( $D$. seguine) que é também uma das mais perigosas e comuns (RODRIGUEZ et al., 2014).

O reconhecimento de um quadro de intoxicação pode ser difícil, pois como afirmam Teixeira e Lima (2011), alguns fatores como cultura e a falta de conhecimento por parte da população podem dificultar o diagnóstico e tratamento da pessoa acidentada por vegetais tóxicos.

Foi perguntado aos gestores se os alunos têm o hábito de tocar nas plantas, onde $57 \%$ responderam que sim, e $43 \%$ responderam que não. Porém, de acordo com os gestores ainda não há nenhum relato de ocorrência de intoxicação causado por alguma planta tóxica nas escolas. Rangel (2000) aponta que é necessário um conhecimento sobre plantas tóxicas por parte do público, no intuído de identificá-las para saber como agir em caso de acidente, principalmente quando este envolve as crianças, e que tais plantas não devem ser removidas do ambiente, devendo-se ensinar à criança que essas plantas não são alimentos.

\section{Plantas ornamentais tóxicas encontradas nas escolas}

Nas escolas pesquisadas foram encontradas e identificadas treze espécies de plantas ornamentais tóxicas, representando cinco famílias botânicas (quadro 02). 
QUADRO 2. Lista de plantas tóxicas encontradas nas escolas de alguns bairros da cidade de Codó - MA, em níveis de família, nome popular, nome científico, parte tóxica e número de escolas com presenças de plantas.

\begin{tabular}{|c|c|c|}
\hline Família / espécie & Nome popular & Parte tóxica \\
\hline
\end{tabular}

Calotropis procera (Aiton) W.T.Aiton Catharanthus roseus (L.) G. Don Thevetia peruviana (Pers.) K. Schum

\section{Araceae}

Anthurium andraeanum Liden

Dieffenbachia seguine (Jacq.) Schott

\section{Asparagaceae}

Sansevieria trifasciata Prain.

\section{Commelinaceae}

Tradescantia spathacea Sw.

\section{Euphorbiaceae}

Codiaeum variegatum (L.) Rumph. ex A.Juss

Euphorbia tirucalli L. Jatropha gossypiifolia L. Manihot esculenta Crantz Pedilanthus tithymaloides (L.) Poit Ricinus communis L. ciúme

vinca

chapéu-de-Napoleão

antúrio

comigo-ninguémpode

espada -de- são jorge

abacaxi-roxo

mosquito

cachorro pelado pinhão-roxo macaxeira dois-irmãos mamona

$\begin{array}{ll}\text { folhas } & 2 \\ \text { folhas } & 3 \\ \text { toda planta } & 1\end{array}$

toda planta

1

toda planta

4

toda planta 8

seiva

sementes

2

toda a planta 1

sementes/látex 3

raiz e folhas 2

toda a planta 1

sementes 1

As famílias mais representativas foram: a) Euphorbiaceae, com seis espécies: Euphorbia tirucalli (cachorro-pelado), Codiaeum variegatum, (mosquito); Manihot esculenta (macaxeira), Jatropha gossypiipholia (pinhão-roxo); Ricinus communis (mamona) e Pedilanthus tithymaloides (dois-irmãos); e b) Apocynaceae: Catharanthus roseus (vinca), Thevetia peruviana (chapéu-de-napoleão) e Calotropis procera (ciúme).

As espécies mais representativas em números de indivíduos cultivados foram: espada-de-são-jorge ( $S$. trifasciata - Euphorbiaceae) encontrada em oito escolas, e comigo-ninguém-pode ( $D$. seguine - Araceae) em quatro escolas.

Bochner et al. (2013), pesquisando escolas públicas do Rio de Janeiro, constataram que de um total de 69 escolas, em 58 ocorrem a presença de ao menos 
uma planta tóxica, onde foram identificadas 23 espécies tóxicas. Entre elas, $S$. trifasciata (espada-de-são-jorge) e $D$. seguine foram as que apresentaram um maior número de indivíduos dentro dos espaços escolares; com registro de um caso de intoxicação por planta. Igualmente, segundo Silva et al. (2017), essas duas espécies foram as mais ocorrentes em escolas do município de Vassouras/RJ.

Também, D. Seguinte foi a mais encontrada em um levantamento em 34 escolas estaduais de Campina Grande, PB (FOOK et al., 2012). Carneiro (2014), fazendo um levantamento de plantas tóxicas em quatro escolas municipais do Município de Coelho Neto - MA, também constatou a presença de três espécies com princípios tóxicos, entre elas a espada-de-são-jorge ( $S$. trifasciata), comigo-ninguémpode ( $D$. seguine) e chapéu-de-napoleão ( $T$. peruviana).

Sousa (2014), registrou a presença de sete espécies de plantas tóxicas nas escolas do Município de Buriti-Maranhão, sendo que três destas espécies pertencem a família Araceae: Comigo-ninguém-pode ( $D$. seguine), tinhorão (Caladium bicolor) e Antúrio (Anthurium andraeanum). Identificou três espécies da família Euphorbiaceae: pinhão-roxo (Jatropha gossypiipholia), pinhão-bravo (Jatropha podagrica), coroa-decristo (Euphorbia milii), e uma espécie da família Asparagaeae: espada-de-são-jorge (Sansevieria trifasciata).

Esses resultados reforçam a importância do conhecimento sobre espécies tóxicas nas escolas, e assim como sugerem Fook et al., (2014) pode, em muito, auxiliar os educadores na tomada de decisões que visem a prevenção de acidentes ao possibilitar que as informações sobre a toxicologia de plantas sejam mais específicas e direcionadas àquelas espécies com as quais a comunidade escolar tem uma possibilidade efetiva de contato.

\section{CONCLUSÕES}

Todas as espécies listadas nesse estudo apresentam um potencial toxicológico considerável, visto que são espécies de ampla ocorrência em áreas escolares. Estão sempre relacionadas aos casos mais comuns de intoxicação com plantas, e são de fácil acesso aos alunos, principalmente crianças, que correm riscos ao manusear ou levar partes desses vegetais à boca.

Sobre o conhecimento das plantas tóxicas, o estudo revelou que há pouca informação sobre o tema, pois alguns entrevistados demonstraram conhecer as plantas tóxicas apenas por nomes, mas não conseguem identificá-las visualmente, o que pode aumentar os riscos de ocorrência de intoxicação nesses espaços escolares onde essas plantas ocorrem. Portando faz-se necessário a elaboração de projetos que incluam no currículo escolar temas que abordem os vegetais tóxicos.

\section{AGRADECIMENTOS}

Os autores agradecem aos diretores das escolas participantes pela permissão para a realização da pesquisa, bem como a todos que responderam os questionários.

\section{REFERÊNCIAS}

ALVES, R. B. S.; LIMA, F. A.; COUTINHO, M. S.; de MENESES, A. B.; LIMA. E. L. M.; MEIRA, A. M. B.; ARAÚJO, C. R. F.; MARIZ, S. R. Plantas ornamentais x Plantas tóxicas: prevenção de acidentes com crianças. Revista Ciência em Extenção, v. 12, 
n. 3, p. 79-87, 2016. Disponível em:<http://ojs.unesp.br/index.php/revista_proex/ article/view/1303/1253>.

ARAÚJO, A. M. R.; PINHEIRO, L. S.; FEITOSA, T. K. A.; FLORÊNCIO, T. A.; MORAIS, I. C. O. A importância do conhecimento de plantas ornamentais tóxicas na prevenção de intoxicações. Mostra Científica da Farmácia, v. 5, n. 1, 2018. Disponívelem:<http://publicacoesacademicas.unicatolicaquixada.edu.br/index.php/mo stracientificafarmacia/article/view/2244>. Acesso em: set, 2018.

BAMPI, A.; SCUR, L.; SCOPEL, J. M. Sensibilização Ambiental Sobre a Importância das Plantas no Jardim Botânico de Caxias do Sul. Scientia Cum Industria, v. 2, n.2, p.77-81,2014. Disponível em: <http://www.researchgate.net/publication/277917511>. doi: 10.18226/23185279.v2iss2p77.

BIONDI, D.; LEAL, L.; SCHAFFER, M. Aspectos importantes das plantas ornamentais em escolas públicas estaduais da cidade de Curitiba, PR. Revista Brasileira de Ciências Agrárias, v.3, n.3, p. 267-275, 2008. Disponível em: <http ://www.agraria.pro.br/ojs-2.4.6/index.php?journal=agraria\&page=article\&op=view\& path\%5B\%5D=58\&path\%5B\%5D=109>. doi: 10.5039/agraria.v3i3a325.

BOCHNER, R.; FISZON, J. T.; ASSIS, M. A. Plantas tóxicas ao alcance de crianças: transformando o risco em informação. Rio de Janeiro: Riobooks, ed. 1, 64p, 2013.

BRAGA, K. C.; GIESE, S. S.; PARRY, M. S. Levantamento de plantas tóxicas em escolas urbanas de Ensino Fundamental do município de Altamira-Pará. Biota Amazônia, v. 7, n. 2, p. 53-58, 2017. Disponível em: <https://periodicos.unifap.br/ index.php/biota/article/view/2137>. doi: 10.18561/2179-5746.

CARNEIRO, A. S. Plantas Tóxicas nas Escolas Municipais de Coelho Neto - MA. Trabalho de conclusão do curso de Licenciatura Plena em Biologia da Universidade Estadual do Maranhão (UEMA), Coelho Neto, 2014, 39p.

BYNG, J. W.; CHASE, M. W.; CHRISTENHUSZ, M. J. M.; FAY, M. F.; JUDD, W. S.; MABBERLEY, D. J.; et al. An update of the Angiosperm Phylogeny Group classification for the orders and families of flowering plants: APG IV. Botanical Journal of the Linnean Society, v. 181, n. 1, p. 1-20, 2016. Disponível em: <http://reflora.jbrj.gov.br/downloads/2016_GROUP_Botanical\%20Journal\%20of\%20th e\%20Linnean\%20Society.pdf>. doi: doi.org/10.1111/boj.12385.

FOOK, S. M. L.; SOARES, Y. C.; ALMEIDA, C. F.; ABRANTES, R. B.; MEIRA, C. M. B. S.; FEITOSA, I. L. F.; MARIZ, S. R. M. Análise da ocorrência de plantas tóxicas em Escolas Estaduais no Município de Campina Grande (PB) como estratégia na prevenção de acidentes. Revista Saúde e Ciência, v. 3, n. 1, p. 44-55, 2014. Disponível em: <http://www.ufcg.edu.br/revistasaudeeciencia/index.php/RSC-UFCG/ article/view/92/81>. Acesso em: nov/2018.

IBGE, 2017. IBGE Cidades. Disponível em: <https: //cidades.ibge .gov .br /brasil /ma /codo/panorama>. Acesso em: agosto/2018. 
MATOS, F. J. A.; LORENZI, H.; SANTOS, L. F. L.; MATOS, M. E. O.; SILVA, M. G. V.; SOUZA, M. P. S. Plantas Tóxicas: Estudo de fitotoxicologia Química de Plantas Brasileiras. São Paulo: Instituto Plantarum, 1 edição, 256p, 2011.

RANGEL, M. S. A. Guia prático para identificação de algumas plantas tóxicas em jardins. Aracaju: Embrapa Tabuleiros Costeiros (Documentos 16), 10p, 2000. Disponível em: <https://www.embrapa.br/busca-de-publicacoes/-/publicacao/370872/ guia-pratico-para-identificacao-de-algumas-plantas-toxicas-em-jardins>.

RODRIGUEZ, L. T.; MELLO, L. A.; GASPARETTO, M.; FILHO, W. J. M. Plantas Ornamentais Tóxicas Ocorrentes no Instituto Federal Catarinense Campus Camboriú. V FICE - Feira de Iniciação Cientifica e de Extensão do Instituto Federal Catarinense, Campus Camboriú, 2014. Disponível em: <http :// www . camboriu.ifc.edu.br/vfice2014/anais/uploads/trab9.pdf>.

SILVA, R. P. P.; ANTÔNIO, S. A.; CAVALCANTE, J. J. V.; MORAES, S. R. Plantas tóxicas em escolas e suas implicações legais em casos de intoxicação. Revista Semioses, v. 11, n. 04, 2017. Disponível em: <http://apl.unisuam.edu.br/revistas/ index.php/Semioses/article/view/1981996X.2017v11n4p21/959>. doi: 10.15202/1981 996x.2017v11n4p21.

SINTOX. 2016. Sistema Nacional de Informações Tóxico-Farmacológicas. Disponível em: <https://sinitox.icict.fiocruz.br/>. Acesso em: nov/2018.

SOUSA, S. J. Levantamento de Plantas Tóxicas em Escolas Públicas do Município de Buriti Maranhão. Monografia apresentada ao Programa Darcy Ribeiro da Universidade Estadual do Maranhão (UEMA), Coelho Neto, 40 p. 2014.

TEIXEIRA, J. B. P.; LIMA, A. A. Plantas Ornamentais Tóxicas: Prevenção de Acidentes. 2011. Universidade Federal de Juiz de Fora, PROPLAMED/TNC (UFJF). Disponível em: <http://www.gege.agrarias.ufpr.br/plantastoxicas/arquivos/euphorbia \%20pulcherrima.pdf>. Acesso em: set/2018.

THE PLANT LIST. 2018. A working list of all plant species. Disponível em: <http:// www.theplantlist.org/>. Acesso em: maio/2018.

TROPICOS. 2018. Missouri Botanical Garden. Disponível em: <http://www. tropicos.org/>, 2018. Acesso em: maio/2018. 